\title{
Technical Improvements on Mobile App Based Taxi Dispatching System
}

\author{
Wenjie Chen \\ Shanghai Key Laboratory of Trustworthy Computing \\ East China Normal University \\ Shanghai, China \\ wjchen@sei.ecnu.edu.cn
}

\begin{abstract}
Current prevalent mobile apps on taxi dispatching help drivers and passengers find each other, which improves the efficiency of booking a taxi compared to traditional mode. However, there are still some critical issues remained or newly introduced, especially on accuracy, equality, safety and honesty. This paper proposes several technical improvements to solve them. What's more, by exploiting more location and other information from the driver and passenger, some additional improvements on route, taximeter and taxi pooling are discussed.
\end{abstract}

Keywords- taxi dispatching system; mobile app; GPS; taxi pooling

\section{INTRODUCTION}

Taxi service is a vital part of urban transportation. How to improve the efficiency is a hot topic in this area. With a GPS terminal installed, a taxi can be located and its trajectory can be record for further analysis and research.

Phithakkitnukoon [1] presented a predictive model for the number of vacant taxis in a given area based on time of the day, day of the week, and weather condition. Li [2] developed a method to represent the Passenger-Finding Strategies based on one year data of all taxis in Hangzhou, China, which can guide taxi drivers to perform better. Powell [3] improved profitability offering location suggestions to taxicab drivers, based on profitability information using historical GPS data, which can reduce overall cruising time. Zhang [4] proposed a method to detect anomalous behavior, such as driving fraud. Ge [5] developed a taxi business intelligence system based on the trace of taxis. Veloso [6] studied the urban traffic by taxi traces data. Yuan $[7,8]$ found out the practically fastest route for a particular car at a given departure time by analyzing a large number of taxi trajectories.

However, these researches are all based on the view of taxi, while the factor of the passenger is omit. With the development of IT technology, a mini-size GPS module can be integrated into a smart phone, so the passenger can be located as well as the taxi, which opens a new era for taxi industry. Chen [9] proposes a dispatching system based on dual locations.

In the industry, mobile app based taxi dispatching systems booms these days, such as Hailo in UK [10], or KuaiDi [11] and DiDi [12] in China. With GPS-enabled smart phones, the apps help the taxi drivers and the passengers know the location of each other, and contact each other directly. This makes it easy to book a taxi. For driver, it reduces the taxi vacancy rate; for passenger, it reduces the waiting time. This improves the success rate of "taking taxi", especially in the remote area.

In China, the competition among the apps focuses on the e-pay section. The main features of different apps are similar. However, there are still some problems unsolved. What's worse, new issues are introduced by these apps. These issues provoke some opposition by the public. So it is important to improve these apps to solve them, or they may be limited by the urban local government in the near future.

\section{CURRENT MODES AND ISSUES}

Traditionally, a passenger call a taxi through two ways: "hail a taxi by the roadside" or "book a taxi via the call center". "Hail" is quite convenient but low efficient, since the taxi and the passenger don't foresee each other in advance. "Call center" is handled by operator manually, so the passenger's information is delayed and fallible.

As an improvement method, most current taxi apps follow the scenario as Fig. 1 illustrated. Both the passenger and the taxi driver have a smart phone with respective app installed. When the passenger wants to take a taxi, he sends a booking request to the server, and then the server will broadcast the request to the taxis nearby (typically around 3 kilometers). If any driver wants to carry this passenger, he should click an "Accept" button as soon as possible. Only the first driver who clicked the button will get the deal successfully. Then the passenger and the driver will make a direct call to exchange detailed information. Finally the taxi drives to the passenger and picks him up.

This mode elevates the opportunity for both the passenger and the driver. However, there are still some issues left or even newly introduced among the following aspects:

1) Accuracy. The request is not served accurately between the demander and the best appropriate provider. The booking request message was broadcast to all the taxis around the passenger, no matter it is "vacant" or "occupied", or whether the driver would rather come or not. Although the passenger's app shows "There are hundreds of taxis around you", but maybe no one will come, especially in the rush hour. It is a terrible experience.

2) Safety. Since the booking request message was broadcast to all the taxis around the passenger, no matter it 
is "vacant" or "occupied", the driver will be flood with the superfluous messages. It will affect the driver's attention. So it is hazardous. On the second hand, a driver may bid for the next deal far before the current passenger reaching the destination, then it will extend the waiting time of the next passenger.

3) Equality. In most cities of China, drivers do not have the right to refuse a hire based on destination for the sake of equality. However, these apps asked the passenger to announce the destination while booking, so the driver can select a "good" business while refuse a "not good" business. The rule of "equality" was broken.

4) Honesty. Currently in China, the app operating companies reward the driver and the passenger who use their apps. Some drivers may cheat for the subsidy with fake transaction. Even the subsidy is canceled in future, fake transaction may be still active for credit reward, good comment and so on.

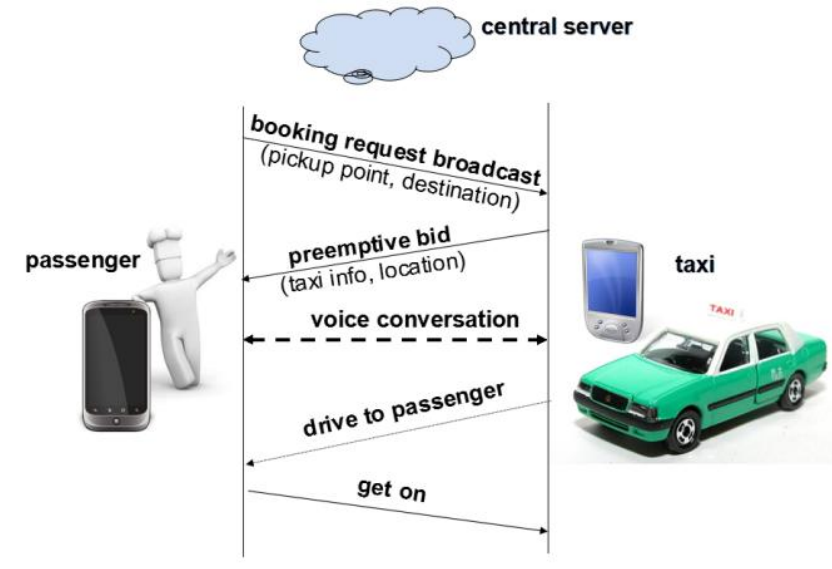

Figure 1. A typical scenario of current taxi app

\section{SOLUTIONS}

\section{A. Cause and solution}

The key point of issues mentioned above is the mode and scenario of "passenger broadcast the request, driver bid for it". Therefore, there are too much superfluous messages sent to the taxis, so the accuracy problem and safety problems occurs. If we let the passenger select the driver, the first 3 mentioned issues will be resolved.

In our new system, the passenger can select an incoming vacant taxi proactively. In order to do this, the system should know the vacancy status and the driving direction of the taxis. We will discuss it soon.

1) However, the manual selection by passenger may be unfair for driver. So, matching by system automatically seems better. When the passenger send a booking request, the system analyzes the request and select an optimized taxi best for all the conditions, send the request only to the specified taxi. This means use unicast instead of broadcast. Whether the driver can refuse or not is decided by the policy of local administration. If the first driver refused, the system may send to the next optimized driver. In order to avoid refusing by destination, the message send to the driver does not include the destination information until the passenger get in.

\section{B. Key Technology}

It is easy to detect the driving direction from GPS signal. Let's see how to detect the vacancy status.

1) Normally, The driver will switch off the "for hire" lamp after passenger getting on. We can transmit signal of the switch to the mobile app via Bluetooth or other similar communication channel.

2) On the contrary, we can also add a button of "picked up" on the driver app. When the passenger was picked up, driver clicks this button, and the "for hire" lamp will be switched off. Technically, it is similar to control the top light to tell the roadside passenger the taxi is reserved as long as the driver accept the deal successfully to avoid a refusal complaint.

3) Both of the above methods need refit the existent hardware. However, we can detect the "vacancy status" by using the GPS positioning function of both the driver side and passenger side. The algorithm is illustrated in Fig.2. The system monitors the driver's position $(p(d r v, t))$ as well as the passenger's $(p(p s g, t))$ continuously. When the driver and the passenger meet $(<\varepsilon)$ and move together long enough $(>\Delta)$, that means the passenger was picked up successfully. It is similar to judge whether the passenger is get off the taxi, mostly after paid. Then the vacancy status of a taxi is obtained.

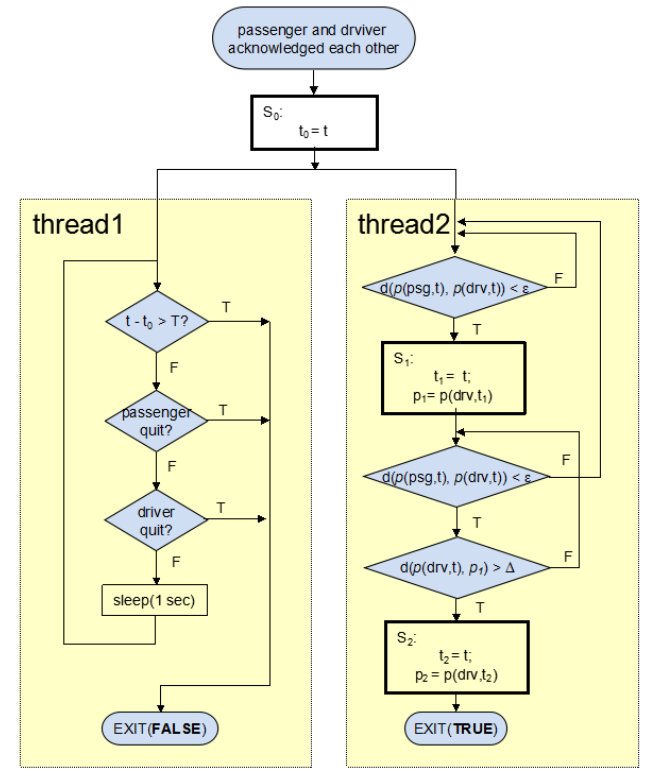

Figure 2. Algorithm to find whether passenger get on or not.

Note:

$\boldsymbol{t}$ is present time, which is transient.

$p(p s g, t)$ is passenger's position on $t . p(d r v, t)$ for driver; $\boldsymbol{d}(\mathbf{p} \mathbf{1 , p 2 )}$ is the distance between $\mathrm{p} 1$ and $\mathrm{p} 2$.

$\varepsilon, \boldsymbol{T}, \Delta$ is the system parameter, which could be set by app user. $\varepsilon$ is the error range for GPS positioning, typically 10 meter.

$\boldsymbol{T}$ is the maximum waiting time, typically 10 second.

$\Delta$ is the minimal distance criteria, typically 200 meter. 
From above scenario, it is obvious that the pick-up and drop-off is detected by the system automatically, so it is impossible for fake transaction. Then the honesty issue is solved as well.

\section{FURTHER IMPROVEMENT}

Current taxi app start with the booking request, end with get-on acknowledgment. The data of location and track of both the taxi and the passenger have not fully exploited. In fact, during and after driving journey, the system can also play the important role. Let's improve it in the following aspects.

\section{A. Route}

Among the taxi complaint cases, overcharging by detour is notable. This issue can be solved easily in our new solution. After receiving the booking request with the information of pickup and destination, the server computes 1 to 3 suggested routes, according to the conditions such as distance, convenience, estimated time, estimated fare and current road conduction. The passenger can choose one route. Both the passenger and the driver will display this route on their app. If the driver is not familiar with the route, voice navigation can be turned on to help him. After reaching the destination, the passenger can compare the real trajectory with the planned route. It can diminish the detour complaint or doubt.

\section{B. App taximeter}

Currently, passenger pays the taxi fare according to the display of the taximeter. However, the taximeter could be tampered by the driver to obtain illegal extra income, such as modifying the $\mathrm{k}$-value (the scale to calculate the mileage based on pulse numbers), adding pulse-generator, and so on. By the way, upon the price was adjusted, or some functions need to be adjusted due to local government's requirement, the taxi have to be driven to the garage for modification.

In principle, the taximeter calculates the fare based on the mileage and time. The time is easily to record. The mileage can be computed via these ways:

1) Fetch the mileage information from ECU (Electronic Control Unit) via OBD interface. This needs an OBD terminal as an extra hardware.

2) Fetch the information from the GPS message. In order to improve the accuracy of mileage, the distance should be calculated frequently in the terminal (mobile phone) instead of transmit the position to the server and calculate there. The challenge is that the GPS signal may be weak or even disappeared in some situations such as in the tunnel or under the viaduct or elevated road. Fortunately, the taxi route is almost fixed and can be planed or estimated so the lost segment is easy to be fixed. And a deviation less that $5 \%$ is acceptable practically. Since the app running in both the driver side and passenger side and monitored by the server, it is infeasible to tamper something. With a app-taximeter, the in-car taximeter could be removed.

\section{Taxi-calling pole}

Generally speaking, taxi service is a universal service. The mobile app is popular among the young people. However, it may be too complicated for those old people. So the app will reduce the availability for the aged. In order to help the aged, the people without smart phone and etc., we can set up a taxi pole on the roadside of some necessary place, such as the market, the residential village and so on. The pole has a call button. If someone wants to take a taxi, he can press the button, then a booking request message will be send out, just the same as the passenger app can do. An ID card reader may be attached to avoid mistakenly touch maliciously or accidentally. The invoice of historical transactions can also be printed by the pole if app-taximeter is adopted.

\section{Taxi pooling}

Taxi pooling means carrying at the same time a number of passengers who did not know each other. Santi [13] introduced the notion of shareability network and applied classical methods from graph theory to solve the taxi pooling problem in a provably efficient way. Generally speaking, taxi pooling saves the social resource, reduces the passenger's cost, and raises the driver's income relatively. Therefore, some cities such as Beijing encourage taxi pooling.

However, taxi pooling is not well accepted in practice, and it was forbidden in some cities, such as Shanghai. The reasons include:

1) Lack of information. The driver and the different passengers don't know each other's destination and willing. It is annoying for both the driver and the passenger to ask "would you like to pool?"

2) Safety. If the passengers are strange to each other, it is hard to build trust among them.

3) How to share the fare? In some situation, the driver will charge all the passengers the full fare. The passengers take no benefit from pooling, so they would rather not to pool. Even the driver would like to give some discount, but how to count?

4) Detour. The passengers may worry about the extra mileage and time on pooling comparing to their respective optimized route.

5) Willing. Maybe the passenger waiting a taxi would rather to pool since it is hard to take a taxi, but the passenger already inside the taxi would rather not for safety, for comfort or for any other concern.

We propose a taxi pooling solution based on the sufficient information. As an improvement, the booking request has an option of "I am willing to pool". If the first passenger checked this option, then after the request was acknowledged, the driver is allowed to answer another booking request during the journey. Of course, only the request from the passenger who also checked the "carpool" option will reach this taxi. The system will analyze the new passenger's request and send the message only to the taxi on the same way, without detour for both the first passenger and the second passenger. A third passenger is also feasible based on the same principle. 
In order to improve the safety, an additional condition can be attached to the "willing" option, such as "I am willing to pool only if other passenger has taken taxi for more than 5 times in this city". The condition may vary according to different demands.

How to share the fare is another key problem of pooling. Beijing has already published a plan, but it only suits for passengers start from the same point. In our solution, it is not necessary. We keep two principles to let all the passengers and driver take advantage from it:

1) Each passenger shares the common mileage, and pay for his own mileage.

2) The driver charges an extra ratio of fee (pooling fee ratio) for shared mileage, more passengers shared, higher the ratio.

For example, if there are 3 passengers: $\mathrm{A}, \mathrm{B}$ and $\mathrm{C}$. When A get off, the taximeter display 10 yuan. And B-20, $C$-30. Let's see how much should $\mathrm{A}, \mathrm{B}$ and $\mathrm{C}$ pays respectively $(f(A), f(B)$, and $f(C))$, as well as how much will the driver earns ( $g($ driver $))$.

The pooling fee ratio should be set reasonably and fairly, which balances between the benefit of the passengers and the driver. Here we set the ratio as $160 \%$ for 3 passengers and $130 \%$ for 2 passengers.

Then,

$$
\begin{aligned}
& f(A)=10 / 3 * 160 \%=5.33 \\
& f(B)=5.33+(20-10) / 2 * 130 \%=11.83 \\
& f(C)=11.83+30-20=21.83 \\
& g(\text { driver })=f(A)+f(B)+f(C)=39.00
\end{aligned}
$$

Therefore, A saves $(10-5.33) / 10=46.7 \%$; B saves $(20$ $11.83) / 20=45.8 \% ; \mathrm{C}$ saves $(30-21.83) / 30=27.2 \%$. And the driver earn $(39-30) / 30=30 \%$ more.

\section{DISCUSSION AND FUTURE WORK}

\section{A. Data Throughput and Energy Consumption}

In order to improve the precision of locating, both the taxi driver and the passenger should keep their smart phone online, which means that the communication channel (WiFi or $3 \mathrm{G}$ ), and the GPS module should keep on during the trip. This will result in the increase of the data throughput and energy consumption. The research is valuable to find a way to control them to reduce the cost and prolong the battery life of the phone.

\section{B. Hardware or Software Improvement}

In this paper, some improvement can be realized by both hardware and software. Generally, the software solution has the advantages because it is easy to gain and maintain, and cheap (or even free) for the users (passengers and drivers). However, some hardware infrastructures are still necessary for special purpose if necessary, such as taxi pole.

\section{Policy of Local government}

In this paper, only the technical issues are discussed. However, the local urban government plays an essential role on the taxi dispatching system. As discussed above, the government should decide the policy on these:
1) Clarify whether it is allowable or not of refusing or selecting passenger.

2) Stipulate the pooling fee ratio by balancing the benefit of drivers and passengers.

3) Clarify whether only the taxis stop on the roadside can accept the request (for safety), or the driver can accept the next business when the current destination is approached soon (for efficiency).

\section{CONCLUSION}

In this paper, we proposed a solution to improve current mobile app based taxi dispatching system technically. We compute the taxi vacancy status and direction from the passenger and driver's relative location. Then we can solve the issues remains in current mobile app, such as accuracy, equality, safety and honesty. Some more aspects are discussed for further improvement, such as route, taximeter and carpooling.

\section{ACKNOWLEDGMENT}

This work was supported by "The Special Funds for the Development of the Internet of Things of China (2013092)".

\section{REFERENCES}

[1] S. Phithakkitnukoon, M. Veloso, C. Bento, A. Biderman, and C. Ratti, "Taxi-aware map: Identifying and predicting vacant taxis in the city,": Springer, 2010, pp. 86-95.

[2] B. Li, D. Zhang, L. Sun, C. Chen, S. Li, G. Qi, and Q. Yang, "Hunting or waiting? Discovering passenger-finding strategies from a large-scale real-world taxi dataset,", 2011, pp. 63-68.

[3] J. W. Powell, Y. Huang, F. Bastani, and M. Ji, "Towards reducing taxicab cruising time using spatio-temporal profitability maps,": Springer, 2011, pp. 242-260.

[4] D. Zhang, N. Li, Z. Zhou, C. Chen, L. Sun, and S. Li, "iBAT: detecting anomalous taxi trajectories from GPS traces," in Proceedings of the 13th international conference on Ubiquitous computing, Beijing, China, 2011, pp. 99-108.

[5] Y. Ge, C. Liu, H. Xiong, and J. Chen, "A taxi business intelligence system," in Proceedings of the 17th ACM SIGKDD international conference on Knowledge discovery and data mining, San Diego, California, USA, 2011, pp. 735-738.

[6] M. Veloso, S. Phithakkitnukoon, C. Bento, N. Fonseca, and P. Olivier, "Exploratory study of urban flow using taxi traces,", 2011.

[7] J. Yuan, Y. Zheng, C. Zhang, W. Xie, X. Xie, G. Sun, and Y. Huang, "T-drive: driving directions based on taxi trajectories,", 2010, pp. 99-108.

[8] J. Yuan, Y. Zheng, X. Xie, and G. Sun, "T-Drive: Enhancing driving directions with taxi drivers' intelligence," Knowledge and Data Engineering, IEEE Transactions on, vol. 25, pp. 220-232, 2013-01012013.

[9] W. Chen and Z. Chai, "A Dual Locations Based Taxi Dispatching System," in ICECC 2012, ZhouShan, China, 2012.

[10] hailo, http://hailocab.com

[11] KuaiDi, http://www.kuaidadi.com/

[12] DiDi, http://www.xiaojukeji.com/

[13] P. Santi, G. Resta, M. Szell, S. Sobolevsky, S. Strogatz, and C. Ratti, "Taxi pooling in New York City: a network-based approach to social sharing problems," arXiv preprint arXiv:1310.2963, 2013 01-012013. 